\title{
A particle swarm approach to solve vehicle routing problem with uncertain demand: A drug distribution case study
}

\author{
Babak Farhang Moghadam $^{\mathrm{a}^{*}}$ and Seyed Mohammad Seyedhosseini ${ }^{\mathrm{a}}$ \\ ${ }^{a}$ Department of Industrial Engineering, Iran University of Science and Technology, Narmak, Tehran Iran
}

\section{A R T I C L E I N F O}

Article history:

Received 1 January 2010

Received in revised form

10 March 2010

Accepted 1 April 2010

Available online 7 April 2010

Keywords:

Meta-heuristic, PSO, VRP

Taguchi method

Robust Optimization

\begin{abstract}
A B S T R A C T
During the past few years, there have tremendous efforts on improving the cost of logistics using varieties of Vehicle Routing Problem (VRP) models. In fact, the recent rise on fuel prices has motivated many to reduce the cost of transportation associated with their business through an improved implementation of VRP systems. We study a specific form of VRP where demand is supposed to be uncertain with unknown distribution. A Particle Swarm Optimization (PSO) is proposed to solve the VRP and the results are compared with other existing methods. The proposed approach is also used for real world case study of drug distribution and the preliminary results indicate that the method could reduce the unmet demand significantly.
\end{abstract}

(c) 2010 Growing Science Ltd. All rights reserved.

\section{Introduction}

During the first decade of the new millennium, there has been an increasing trend on oil price which result to an increase to cost of supply chain and logistics. Therefore, there have been tremendous efforts to develop realistic problems in supply chain management and logistics to reduce the associated cost as much as possible (Clark and Scarf, 1960; Graves et al., 1993; Garaix et al., 2010; Andersson et al., 2010). One of the primary concerns on most of the real world supply chain problems is the complexity of the resulted mathematical formulations. In fact, in many real world case studies of supply chain problems, we are normally faced with a Mixed Integer Problem where the number of binary variables exceeds to over a few hundred even for small case studies. Therefore, we are unable to find the optimal solution in a reasonable amount of time. The problem is more crucial when we intent to solve the problem almost in real time. On the other hand, we may not be interested in having an exact optimal solution and this could motivate us to look for near optimal solutions. There are tremendous efforts to develop some heuristic or meta-heuristic methods to find the near optimal solutions. There have been varieties of meta-heuristic methods introduced in the literature such as Genetic Algorithm, Ant Colony Optimization, Neural Network, Particle Swarm Optimization (PSO), etc. (Kennedy \& Eberhart, 1995) are believed the first people who introduce the concept of PSO. PSO is a type of swarm intelligence provides insights into social behavior, as well as contributing to engineering applications. Ai and Kachitvichyanukul (2009a) develop a PSO for VRP with simultaneous pickup and delivery and they compared the performance of their method with other existing meta-heuristic methods using some benchmark problems. Ai and Kachitvichyanukul (2009b) use similar PSO for capacitated VRP and report some promising results. PSO has been widely implemented for many logistics problems (Ai and Kachitvichyanukul, 2009b; Ai and Kachitvichyanukul, 2008; Tao et al., 2008; Chen et al., 2006). Önüt et al. (2008), for instance, use PSO algorithm for the multiple-level warehouse layout design problems. Shi et al. (2007) are the first people who successfully applied PSO for Traveling Salesman Problem (TSP). Since VRP is an extension of TSP, we may also use PSO for a selected form of VRP. Marinakis and Marinaki (2010) use a hybrid PSO for TSP where a 
new hybrid algorithmic nature inspired approach based on PSO, Greedy Randomized Adaptive Search Procedure (GRASP) and Expanding Neighborhood Search Strategy (ENS) is developed. They test the performance of their proposed method on numerous benchmark problems from TSPLIB and claim some satisfactory results. Many researchers suggest employing a hybrid strategy, which embeds a local optimizer in between the iterations of the meta-heuristics (Chen et al., 2006). One of the primary concerns on traditional VRP is that all input parameters are assumed to be deterministic (Bertsimas, 1992). Therefore, a small perturbation on input data could result some impractical solutions. Jaillet and Odoni (1988) proposed some heuristics to solve the probabilistic VRP. Dror (1993) presented SVRP by Markov Decision Process. Golden et al. (1979) developed a chance constrained programming model for VRP with stochastic demand. Stewart et al. (1983) presented some computational results over the work of Golden and Yee (1979). In this paper, we study a special form of VRP where demand is contaminated with perturbation. We assume that the distribution of demand is unknown but we consider it in symmetric interval. The resulted model is formulated as mixed integer nonlinear problem and there are literally hundreds of binary variables involved making it difficult to solve the problem for the optimality. Therefore, we use PSO to determine the near optimal solutions and compare the results with other meta-heuristic methods. This paper is organized as follows. In section 2, we explain the necessary notations and problem statement, PSO explanation and the result of the implementation of our PSO are compared with other existing meta-heuristic. Section 3 is devoted to a real world case study of VRP in drug industry. Finally, conclusion remarks are given at the end to summarize the contribution of the paper.

\section{Problem statement}

VRP is normally a combinatorial optimization problem seeking to serve a number of customers with a fleet of vehicles. Proposed by Dantzig and Ramser (1959), VRP is an important problem in the fields of transportation, distribution and logistics. In a typical VRP we have a central depot where different vehicles are responsible to deliver goods for various customers and the primary objective is to minimize the total transportation cost. Since VRP is an NP-Hard problem (Lenstra \& Rinnooy, 1981), there have been tremendous efforts to use meta-heuristic methods such as PSO to find a near optimal solution. PSO itself cannot solve a VRP directly because it needs a decoding procedure. Ai and Kachitvichyanukul (2009a) introduces different decoding algorithms and Ai and Kachitvichyanukul (2009b) determine SR_2 as the best one to solve CVRP among the other methods In the following we present the algorithm and related decoding methods.

$$
\begin{aligned}
& \alpha \quad \text { iteration index, } \alpha=1,2,3, \ldots, T \\
& k \quad \text { particle index, } k=1,2,3, \ldots, K \\
& S \quad \text { dimension index, } s=1,2,3, \ldots . . S \\
& u \quad \text { uniform random number in the interval }[0,1] \\
& w(\alpha) \quad \text { inertia weight in the } \alpha \text { th iteration } \\
& v_{k s}(\alpha) \text { velocity of the } k \text { th particle at the } S \text { th dimension in the } \alpha \text { th iteration } \\
& \theta_{k s}(\alpha) \text { position of the } k \text { th particle at the } S \text { th dimension in the } \alpha \text { th iteration } \\
& \eta_{k s} \quad \text { personal best solution (pbest) of the } k \text { th particle at the } S \text { th dimension } \\
& \eta_{g s} \quad \text { global best solution (gbest) at the sth dimension } \\
& \eta_{k s}^{K} \quad \text { local best solution (kbest) of the } k \text { th particle at the } k \text { th dimension } \\
& \eta_{k s}^{N} \quad \text { near neighbor best solution (nbest) of the } k \text { th particle at the } S \text { th dimension }
\end{aligned}
$$


$C_{p} \quad$ personal best solution acceleration constant

$C_{g} \quad$ global best solution acceleration constant

$C_{k} \quad$ local best solution acceleration constant

$C_{n} \quad$ near neighbor best solution acceleration constant

$\theta_{\max }$ maximum position value

$\theta_{\min } \quad$ minimum position value

$\Theta_{k} \quad$ vector position of the $k$ th particle

$\Omega_{k} \quad$ vector velocity of the $k$ th particle, $\left[\omega_{k 1}, \omega_{k 2}, \ldots \omega_{k S}\right]$

$\eta_{k} \quad$ vector personal best solution of the $k$ th particle.

$\eta_{g} \quad$ vector global best solution

$\eta_{k}^{K} \quad$ vector local best solution of the $k$ th particle,

$R_{k} \quad$ the $k$ th set of vehicle route

$Z\left(\Theta_{k}\right)$ fitness value of $\Theta_{k}$

FDR fitness distance ratio

\subsection{Algorithm}

1. Initialize $K$ particles as a swarm, generate the $k$ th particle with random position $\Theta_{k}$ in the range of [ $\theta_{\text {min }}$, $\left.\theta_{\max }\right]$, velocity $\Omega_{k}=0$ and personal best $\eta_{k}=\Theta_{k}$ for $k=1 \ldots K$, Set iteration $\alpha=1$.

2. For $k=1 \ldots K$, decode $\Theta_{k}(\alpha)$ to a set of vehicle route $R_{k}$.

3. For $k=1 \ldots K$, compute the performance measurement of $R_{k}$, and set this as the fitness value of $\Theta_{k}$, represented by $Z\left(\Theta_{k}\right)$.

4. Update pbest: For $k=1 \ldots K$, update $\eta_{k}=\Theta_{k}$, if $Z\left(\Theta_{k}\right)<Z\left(\eta_{k}\right)$.

5. Update gbest: For $\mathrm{k}=1 \ldots \mathrm{K}$, update $\eta_{g}=\eta_{k}$, if $Z\left(\eta_{k}\right)<Z\left(\eta_{g}\right)$.

6. Update lbest: For $k=1 \ldots K$, among all pbest from $J$ neighbors of the $k$ th particle, set the personal best which obtains the least fitness value to be $\eta_{k}^{K}$.

7. Generate nbest: For $k=1 \ldots K$, and $s=1 \ldots S$, set $\eta_{k s}^{N}=\eta_{o s}$ and maximize fitness-distance-ratio (FDR) for $o=1 \ldots S$. where

FDR is defined as (Veeramachaneni et al, 2003):

$F D R=\frac{Z\left(\Theta_{k}\right)-Z\left(\eta_{o}\right)}{\left|\theta_{k s}-\eta_{o s}\right|}$ where $\quad k \neq o$

8. Update the velocity and the position of each $k$ th particle: 


$$
\begin{aligned}
& w(\alpha)=w(T)+\frac{\alpha-T}{1-T}[w(1)-w(T)] \\
& v_{k s}(\alpha+1)=w(\alpha) v_{k s}(\alpha)+c_{p} u\left(\eta_{p s}-\theta_{k s}(\alpha)\right)+c_{g} u\left(\eta_{g s}-\theta_{k s}(\alpha)\right) \\
& +c_{k} u\left(\eta_{k s}^{K}-\theta_{k s}(\alpha)\right)+c_{n} u\left(\eta_{k s}^{N}-\theta_{k s}(\alpha)\right) \\
& \theta_{k s}(\alpha+1)=\theta_{k s}(\alpha)+v_{k s}(\alpha+1) \\
& {\left[\begin{array}{c}
\text { if } \quad \theta_{k s}(\alpha+1)>\theta^{\max }, \text { then } \\
\theta_{k s}(\alpha+1)=\theta^{\max } \\
v_{k s}(\alpha+1)=0 \\
\theta_{k s}(\alpha+1)<\theta^{\min }, \text { then } \\
\theta_{k s}(\alpha+1)=\theta^{\min } \\
v_{k s}(\alpha+1)=0
\end{array}\right.}
\end{aligned}
$$

9. If the stopping criterion is met, i.e. $\alpha=T$, stop. Otherwise, $\alpha=\alpha+1$ and return to step 2 .

\subsection{Solution representation}

As we already explained, the PSO provides a vector $X_{i}$ and the final optimal solution cannot be derived directly. Therefore we need a decoding approach to allocate the vehicles for each rout and in this paper SR-2 (Ai and Kachitvichyanukul, 2009b) is used to decode PSO final solution to determine the vehicle routs. The solution representation SR-2 consists of $3 \mathrm{~m}$ dimensional particle where each particle dimension is encoded as a real number. All dimensions are related to vehicles, each vehicle is represented by three dimensions: two dimensions for the reference point and one dimension for the vehicle coverage radius. In the GLNPSO algorithm, the fitness function calculates the sum of distances among all routes. However, it cannot create a good balance among various routes. Table 1 summarizes the information of balanced and unbalanced routes of a simple VRP example where the balanced VRP has slightly higher cost but demands are not highly deviated.

Table 1

Data of a conceptual example

\begin{tabular}{lllllllll}
\hline costumer No. & $\operatorname{depot}(1)$ & 2 & 3 & 4 & 5 & 6 & 7 & 8 \\
\hline Longitude & 82 & 82 & 99 & 70 & 80 & 58 & 93 & 62 \\
\hline Latitude & 50 & 90 & 51 & 85 & 20 & 60 & 60 & 45 \\
\hline Demand & 0 & 46 & 46 & 44 & 32 & 10 & 34 & 45 \\
\hline
\end{tabular}

Capacity of vehicle: 100

It is clear that the best solution in Fig. 1, is not robust against the perturbation. In fact, a small change in demand for the route one may exceed the total capacity. In Fig. 2, although the cost increases $2 \%$, all the routes can resist against the demand uncertainty as long as the perturbation is less than $16 \%$. This simple example with 8 nodes and 3 routes is built to demonstrate the consequences of having an unbalanced demand. In real world problem, we may face more sever incidents which could cost us significantly. The following explains the details of the balancing procedure. Let $\widetilde{d}_{i}$ be the demand customer with $\left(d_{i_{-}} \varepsilon d_{i} \leq d \leq d_{i}+\varepsilon d_{i}\right)$ where $\varepsilon$ indicates the perturbation percentage. Therefore we have,

$D_{l} \leq \sum_{i} \widetilde{d}_{i} \leq D_{u}$ 
where $D_{l}$ and $D_{u}$ are the lower bound and the upper bounds for the sum of the real perturbed demands, respectively. Also we have:

$\psi=\left(\operatorname{VAR}\left(\lambda_{v}\right)\right)^{-1}$,

$\lambda_{v}=\Delta \rho_{v}-\phi$

$\phi=\max \left\{\frac{\sum_{v} \Delta \rho_{v}}{n}, \frac{D_{u}-\sum d_{i}}{n}\right\}$,

where $\psi$ represents the changes of unused capacity for route $v$ which includes $(i, j)$ and $\Delta \rho_{v}$ is unused capacity for each rout.

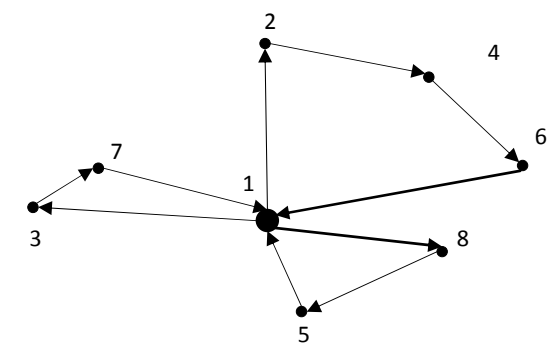

Route 1:2, 4, 6 ; Demand sum: 100

Route $2: 3,7$; Demand sum: 80

Route 3: 5,8 ; Demand sum: 77

Total Cost: 230

Fig. 1. The best solution

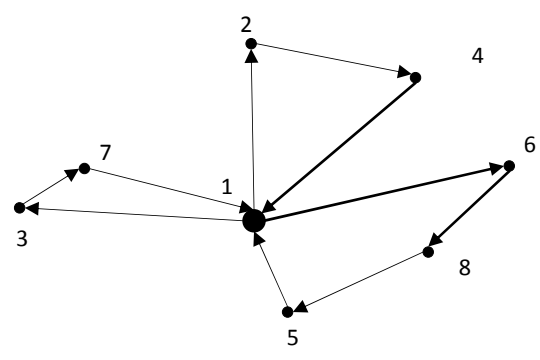

Route 1:2, 4 ; Demand sum: 90

Route 2: 3,7 ; Demand sum: 80

Route 3: 5, 8, 6 ; Demand sum: 87

Total Cost: 235

Fig. 2. The robust solution

The purpose of equations (8) is to make sure that the limit for the remaining capacity of each vehicle does not exceed its upper bound. Equation (3) is normally used to adjust the velocity in PSO. In this paper, we propose a new one for the robust VRP as follows:

$v_{k s}(\alpha+1)=w(\alpha) v_{k s}(\alpha)+c_{p} u\left(\eta_{p s}-\theta_{k s}(\alpha)\right)\left(\psi_{p}\right)+c_{g} u\left(\eta_{g s}-\theta_{k s}(\alpha)\right)\left(\psi_{g}\right)+c_{k} u\left(\eta_{k s}^{K}-\theta_{k s}(\alpha)\right)\left(\psi_{K}\right)+c_{n} u\left(\eta_{k s}^{N}-\theta_{k s}(\alpha)\right)\left(\psi_{N}\right)$.

Table 2 summarizes the results for the implementation of our PSO method and it compares the results with exact robust VRP. It is clear that the optimal solutions for large scale problems are not available due to the complexity of the mathematical model. Therefore, we need to use some techniques to measure the performance of our proposed method when demands are contaminated with perturbation. Let $Z_{U D}$ and $Z_{U R}$ be unmet demand for deterministic and robust, respectively. In order to find $Z_{U D}$, a nominal problem is solved with known demand first and the demands are perturbed with $\left(d_{i_{-}} \varepsilon d_{i}, d_{i}+\varepsilon d_{i}\right)$. Now, the sum of unmet demand is calculated and stored in $Z_{U D} \cdot Z_{U R}$ is also calculated using similar procedure with the proposed PSO. When $Z_{U D}$ is compared with $Z_{U R}$ we may easily find out how effective a robust PSO could perform against the standard PSO when we confront noise in our data. Table 3 summarizes the results of our implementation. As we can observe, when $\varepsilon=.1 \%$ there is no unmet demand for some cases. As $\varepsilon$ increases there is a big gap between the unmet demands for robust and deterministic solutions although the cost of transportation could be a bit worsen. 


\section{A Distribution Case Study}

Behpakhsh Company, as a large wholesaler and distributing agency, produces hygienic products and it is located in Tehran/Iran and some other Iranian cities. This company is presently covering 5700 customers in a vicinity of 90,000 acres $\left(900 \mathrm{~km}^{2}\right)$. As a VRP case study, we have concentrated on only one region of Behpakhsh territory. This region includes 82 customers which Behpakhsh serves them one product type with 100 vehicles. We attempt to find a robust solution to satisfy the demand for Behpakhsh in this region.

Table 2

Proposed PSO qualification against uncertainty

\begin{tabular}{|c|c|c|c|c|c|c|c|c|c|c|c|}
\hline \multirow[b]{2}{*}{ Row } & \multirow[b]{2}{*}{$N$} & \multirow[b]{2}{*}{$Z_{d}$} & \multirow[b]{2}{*}{$Z_{r}$} & \multicolumn{2}{|c|}{$\varepsilon=0.1 \%$} & \multirow[b]{2}{*}{$Z_{r}$} & \multicolumn{2}{|l|}{$\varepsilon=1 \%$} & \multicolumn{2}{|c|}{$\varepsilon=10 \%$} & \multirow[b]{2}{*}{$Z_{U R}$} \\
\hline & & & & $Z_{U D}$ & $Z_{U R}$ & & $Z_{U D}$ & $Z_{U R}$ & $Z_{r}$ & $Z_{U D}$ & \\
\hline 1 & 32 & 784 & 788 & $0.51 \%$ & 0.04 & 788 & 0.51 & 0.09 & 789 & $0.64 \%$ & 0.13 \\
\hline 2 & 33 & 661 & 678 & $2.57 \%$ & 0 & 690 & 4.39 & 0.03 & 691 & $4.54 \%$ & 0.03 \\
\hline 3 & 33 & 742 & 745 & $0.40 \%$ & 0.06 & 747 & 0.67 & 0.06 & 749 & $0.94 \%$ & 0.09 \\
\hline 4 & 34 & 778 & 781 & $0.39 \%$ & 0 & 782 & 0.51 & 0.03 & 784 & $0.77 \%$ & 0.04 \\
\hline 5 & 36 & 799 & 800 & $0.13 \%$ & 0.08 & 800 & 0.13 & 0.08 & 800 & $0.13 \%$ & 0.11 \\
\hline 6 & 37 & 669 & 671 & $0.30 \%$ & 0.03 & 672 & 0.45 & 0.08 & 672 & $0.45 \%$ & 0.08 \\
\hline 7 & 37 & 949 & 951 & $0.21 \%$ & 0 & 952 & 0.32 & 0 & 953 & $0.42 \%$ & 0.05 \\
\hline 8 & 38 & 730 & 733 & $0.41 \%$ & 0.08 & 733 & 0.41 & 0.08 & 735 & $0.68 \%$ & 0.11 \\
\hline 9 & 39 & 822 & 830 & $0.97 \%$ & 0.03 & 833 & 1.34 & 0.03 & 837 & $1.82 \%$ & 0.05 \\
\hline 10 & 39 & 831 & 839 & $0.96 \%$ & 0.06 & 844 & 1.56 & 0.1 & 847 & $1.93 \%$ & 0.13 \\
\hline 11 & 44 & 937 & 939 & $0.21 \%$ & 0.05 & 939 & 0.21 & 0.07 & 940 & $0.32 \%$ & 0.09 \\
\hline 12 & 45 & 944 & 947 & $0.32 \%$ & 0 & 948 & 0.42 & 0.02 & 951 & $0.74 \%$ & 0.07 \\
\hline 13 & 45 & 1146 & 1155 & $0.79 \%$ & 0.04 & 1157 & 0.96 & 0.09 & 1158 & $1.05 \%$ & 0.11 \\
\hline 14 & 46 & 914 & 918 & $0.44 \%$ & 0.02 & 920 & 0.66 & 0.07 & 922 & $0.88 \%$ & 0.11 \\
\hline 15 & 48 & 1073 & 1088 & $1.40 \%$ & 0.06 & 1095 & 2.05 & 0.08 & 1107 & $3.17 \%$ & 0.1 \\
\hline 16 & 53 & 1010 & 1018 & $0.79 \%$ & 0.04 & 1022 & 1.19 & 0.08 & 1025 & $1.49 \%$ & 0.16 \\
\hline 17 & 54 & 1167 & 1176 & $0.77 \%$ & 0.02 & 1176 & 0.77 & 0.06 & 1181 & $1.20 \%$ & 0.07 \\
\hline 18 & 55 & 1073 & 1082 & $0.84 \%$ & 0.02 & 1086 & 1.21 & 0.04 & 1092 & $1.77 \%$ & 0.04 \\
\hline 19 & 61 & 1035 & 1042 & $0.68 \%$ & 0.02 & 1047 & 1.16 & 0.03 & 1053 & $1.74 \%$ & 0.07 \\
\hline 20 & 62 & 1290 & 1307 & $1.32 \%$ & 0.03 & 1315 & 1.94 & 0.05 & 1319 & $2.25 \%$ & 0.06 \\
\hline 21 & 63 & 1315 & 1325 & $0.76 \%$ & 0.06 & 1331 & 1.22 & 0.1 & 1336 & $1.60 \%$ & 0.14 \\
\hline 22 & 63 & 1634 & 1650 & $0.98 \%$ & 0.05 & 1655 & 1.29 & 0.06 & 1667 & $2.02 \%$ & 0.08 \\
\hline 23 & 64 & 1402 & 1415 & $0.93 \%$ & 0.06 & 1415 & 0.93 & 0.06 & 1421 & $1.36 \%$ & 0.08 \\
\hline 24 & 65 & 1177 & 1185 & $0.68 \%$ & 0.08 & 1186 & 0.76 & 0.08 & 1188 & $0.93 \%$ & 0.09 \\
\hline 25 & 69 & 1168 & 1179 & $0.94 \%$ & 0.07 & 1187 & 1.63 & 0.09 & 1189 & $1.80 \%$ & 0.1 \\
\hline 26 & 80 & 1764 & 1781 & $0.96 \%$ & 0 & 1787 & 1.30 & 0.04 & 1799 & $1.98 \%$ & 0.05 \\
\hline
\end{tabular}

\subsection{Data gathering}

The following summarizes the data set and the information needed to solve the robust and the deterministic models.

Demand perturbation: The percentage deviation in customers' demands

Customers' locations: This data is used to specify access paths, which eventually turns out to estimate time distance between each two individual nodes.

Customers' demands: This data varies in different periods of time. They are gathered from marketing department. 
Time distance between the nodes of the network: These data show the length distance between each two nodes.

Since the company only has the time distances between the customers based on the existing paths situations, potential cost coefficients remain uncovered. Moreover, Time and Motion Study of the network would be too costly; therefore, time distance between each two nodes of the fully connected network is calculated. After determining the possible paths, the distance times between each two nodes are calculated based on Table 3 .

\section{Table 3}

Average speeds on the paths according to $V / C$, where $V$ is the average number of vehicles in the path and $C$ is the capacity of the path

\begin{tabular}{ccc}
\hline Paths Categorization & V/C & Speed $(\mathrm{km} / \mathrm{h})$ \\
\hline A & 0.35 & 90 \\
B & 0.55 & 75 \\
C & 0.7 & 60 \\
D & 0.8 & 45 \\
E & 1 & 10 \\
\hline
\end{tabular}

We have performed a survey on the actual information of the orders and demands. Our experiment indicates that when a particular customer is served, he/she may ask more or less than his/her orders. Table 4 summarizes the percentage deviation between the actual purchase and the orders (perturbation percentage). The table also shows the frequency of customers engaged with each perturbation category (customer frequency percentage).

Table 4

Percentage deviation in customers' demands

\begin{tabular}{cccc}
\hline Category & Perturbation (\%) & Range Index & Customer frequency (\%) \\
\hline 1 & $5-10$ & 7.5 & 0.04 \\
2 & $10-15$ & 12.5 & 0.06 \\
3 & $15-20$ & 17.5 & 0.08 \\
4 & $20-25$ & 22.5 & 0.06 \\
5 & $25-30$ & 27.5 & 0.05 \\
6 & $30-35$ & 32.5 & 0.11 \\
7 & $35-40$ & 37.5 & 0.19 \\
8 & $40-45$ & 42.5 & 0.19 \\
9 & $45-50$ & 47.5 & 0.12 \\
10 & $50-55$ & 52.5 & 0.12 \\
11 & $55-60$ & 57.5 & 0.07 \\
12 & $60-65$ & 62.5 & 0.03 \\
\hline
\end{tabular}




\subsection{Robust and deterministic solutions}

In order to have a better understanding of the behavior of the robust model versus the deterministic one, we have solved the case study under both deterministic and robust conditions. Table 5 illustrates the details of the implementations. In this table $Z_{R}$ represents the transportation cost of the robust solution, $Z_{D}$ denotes the transportation cost for the deterministic solution, and $Z_{U D}$ shows unsatisfied demand for the solutions based on deterministic demand, whereas the actual demands are contaminated by perturbation. As it can be observed, an increase in perturbation will result to an increase in $Z_{U D}$, that is, $Z_{U D}$ is sensitive to perturbation percentage.

Table 5

Robust and deterministic solutions for Behpakhsh case study

\begin{tabular}{ccccc}
\hline No & \%Perturbation & $Z_{D}$ & $Z_{R}$ & $Z_{U D}$ \\
\hline 1 & 7.5 & 134 & 134.5 & 0.4 \\
2 & 12.5 & 134 & 134.9 & 1.5 \\
3 & 17.5 & 134 & 135.2 & 2.8 \\
4 & 22.5 & 134 & 135.4 & 4 \\
5 & 27.5 & 134 & 135.55 & 5.3 \\
\hline 6 & 32.5 & 134 & 135.7 & 6.7 \\
\hline
\end{tabular}

\begin{tabular}{ccccc}
\hline NO & \%Perturbation & $Z_{D}$ & $Z_{R}$ & $Z_{U D}$ \\
\hline 7 & 37.5 & 134 & 136.8 & 8.4 \\
8 & 42.5 & 134 & 137.6 & 10.2 \\
9 & 47.5 & 134 & 138.4 & 12.8 \\
10 & 52.5 & 134 & 139 & 15 \\
11 & 57.5 & 134 & 139.5 & 18.3 \\
\hline 12 & 62.5 & 134 & 139.95 & 23.1 \\
\hline
\end{tabular}

\subsection{Cost Analysis}

In Behpakhsh case study, we assume the lost profit of each unsatisfied order product unit is $2 \mathrm{~A}$, and the cost of each vehicle transportation per hour is $5 \mathrm{~A}$, where $\mathrm{A}$ is a classified monitory unit. Table 6 summarizes the costs for the robust and the deterministic solutions. $\operatorname{Cost}_{D}$ and $\operatorname{Cost}_{R}$ are deterministic and robust solution costs, respectively.

Table 6

Cost analysis for Behpakhsh case study

\begin{tabular}{|c|c|c|c|c|c|c|c|c|c|}
\hline & & & & & \\
\hline No & $\%$ Perturbation & $\operatorname{Cost}_{L}$ & $\operatorname{Cost}_{R}$ & $D$ & No. & \%Perturbation & $\operatorname{Cost}_{D}$ & $\operatorname{Cost}_{R}$ & $D$ \\
\hline 1 & 7.5 & 0.8 & 2.5 & -1.7 & 7 & 37.5 & 16.8 & 14 & 2.8 \\
\hline 2 & 12.5 & 3 & 4.5 & -1.5 & 8 & 42.5 & 20.4 & 18 & 2.4 \\
\hline 3 & 17.5 & 5.6 & 6 & -0.4 & 9 & 47.5 & 25.6 & 22 & 3.6 \\
\hline 4 & 22.5 & 8 & 7 & 1 & 10 & 52.5 & 30 & 25 & 5 \\
\hline 5 & 27.5 & 10.6 & 7.75 & 2.85 & 11 & 57.5 & 36.6 & 27.5 & 9.1 \\
\hline 6 & 32.5 & 13.4 & 8.5 & 4.9 & 12 & 62.5 & 46.2 & 29.75 & 16.45 \\
\hline
\end{tabular}

For instance, in the first row, $\operatorname{Cost}_{D}$ is reached by $5 A\left(Z_{r}-Z_{u}\right)$, and $\operatorname{Cost}_{R}$ is calculated by $2 A Z_{U D}$. Eventually, $\mathrm{D}=$ Cost $_{D}-$ Cost $_{R}$ represents the net profit of the robust solution. The experimental results of this work shows that the net profit is synchronized with the perturbation percentage. Therefore, we would gain more benefit with robust solution as the perturbation increases. In this case study, if we use the expected value of the profit, based on Table 10, we get 24.15 which is 12.6 percent of the Company's related income. Note that a ten percent increase in total net profit of publicly traded company can significantly influence the stock 
share's price. The results of the implementation of our proposed method have been used by the company and they have implemented some action plans on their own traditional routes. In a three months period, the transportation cost increased only $2.6 \%$ but the delivery volume of goods was also improved by $11 \%$. Overall, the results significantly made changes on profitability and created a motivation for the management of the company to use this method. Note that the recent increase on energy prices could increase the motivation to use efficient transportation planning.

\section{Conclusions}

We have presented a PSO method for a robust VRP with uncertain demand. A response surface methodology has been used to tune the PSO parameters. The proposed method of this paper has been implemented on some existing data used in the literature. The preliminary results of this study have shown that an efficient and robust routing planning may increase the cost of transportation but it would significantly reduce the unmade demand which could help us penetrate into a wider market. As we have shown for the implementation of our proposed method for a real world case study, a robust VRP plan increases the total transportation expenditure but, at the same time, it can increase the unmade demand notably.

\section{Acknowledgment}

The authors would like to thank the anonymous referees whose comments significantly improved this paper.

\section{References}

Ai, J., \& Kachitvichyanukul, V. (2009.a). A particle swarm optimization for the vehicle routing problem with simultaneous pickupand delivery. Computers \& Operations Research, 36, 1693-1702.

Ai, J., \& Kachitvichyanukul, V. (2009.b). Particle swarm optimization and two solution representations for solving the capacitated vehicle routing problem. Computers \& Industrial Engineering, 56 (1), 380-387.

Ai, J., \& Kachitvichyanukul, V. (2009.c). A Particle Swarm Optimisation for Vehicle Routing Problem with Time Windows. International Journal of Operational Research, 6 (4), 519-537.

Ai, J., \& Kachitvichyanukul, V. (2008). A Study on Adaptive Particle Swarm Optimization for Solving Vehicle Routing Problems. The 9th Asia Pacific Industrial Engineering and Management Systems Conference.

Andersson, H., Hoff, A., Christiansen, M., Hasle, G. \& Løkketangen, A. (2010). Industrial aspects and literature survey: Combined inventory management and routing, Computers \& Operations Research, 37(9), 1515-1536.

Bertsimas, D. J. (1992). A vehicle routing problem with stochastic demand. Operations Research , 40 (3), 574585.

Chen, A., Yang, G., \& Wu, Z. (2006). Hybrid discrete particle swarm optimization algorithm for capacitated vehicle routing problem. Journal of Zhejiang University Science, 7 (4), 607-614.

Clarke, G., \& Wright, J. W. (1964). Scheduling of vehicles from a central depot to a number of delivery points. Operations Research, 12, 568-589.

Dantzig, G. B., \& Ramser, J. H. (1959). The truck dispatching problem. Management Science, 6, 80-91.

Dror, M. (1993). Modeling vehicle routing with uncertain demands as a stochastic program. European Journal of Operational Research , 64, 432-441.

Golden, B. L., \& Yee, J. R. (1979). A framework for probabilistic vehicle routing. AIIE Transactions, 11, 109112.

Graves, S. C., Rinooy Kan, A., \& Zipkin, P. (1993). Logistics of Production and Inventories: Handbooks in OR \& MS (Vol. 4). Elsevier Science.

Garaix, T., Artigues, C., Feillet, D. \& Josselin, D. (2010). Vehicle routing problems with alternative paths: An application to on-demand transportation, European Journal of Operational Research, 204, 62-75.

Jaillet, P., \& Odoni, A. (1988). The Probabilistic Vehicle Routing Problem, in Vehicle Routing; Methods and Studies. In B. L. Golden, \& A. A. Assad, (Eds.) Amsterdam: North Holland.

Kennedy, J., \& Eberhart, R. (1995). Particle swarm optimization. IEEE Int. Conf. Neural Networks, 19421948. 
Lenstra, J. K., \& Rinnooy Kan, A. (1981). Complexity of vehicle routing and scheduling problems. Networks, $11,221-227$.

Marinakis, Y. \& Marinaki, M. (2010). A Hybrid Multi-Swarm Particle Swarm Optimization algorithm for the Probabilistic Traveling Salesman Problem, Computers and Operations Research, 37 (3), 432-442.

Önüt, S., Tuzkaya, R. U., \& Doğaç, B. (2008). A particle swarm optimization algorithm for the multiple level warehouse layout design problem. Computers \& Industrial Engineering, 54 (4), 783-799.

Shi, X. H., Liang, Y. C., Lee, H. P., Lu, C., \& Wang, Q. X. (2007). Particle swarm optimization based algorithms for TSP and generalized TSP. Information Processing Letters, 103 (5), 169-176.

Stewart, W. R., \& Golden, B. L. (1983). Stochastic vehicle routing. European Journal of Operational Research, 14, 371-385.

Tao, Z., Chunmei, Z., Yuejie, Z., \& Chuoya, Y. (2008). A Mixed PSO algorithm for the VRPSPD. Control and Decision Conference, (pp. 4017-4021).

Veeramachaneni, K., Peram, T., Mohan, C., \& Osadciw, L. (2003). Optimization using particle swarm with near neighbor interactions. Genetic and Evolutionary Computation Conference. 